\title{
Optimal operation of parallel dead-end filters in a continuous bio-based process
}

Bähner, F. D.; Santacoloma, P. A.; Huusom, J. K.

Published in:

Food and Bioproducts Processing

Link to article, DOI:

10.1016/j.fbp.2019.02.001

Publication date:

2019

Document Version

Peer reviewed version

Link back to DTU Orbit

Citation (APA):

Bähner, F. D., Santacoloma, P. A., \& Huusom, J. K. (2019). Optimal operation of parallel dead-end filters in a continuous bio-based process. Food and Bioproducts Processing, 114, 263-275.

https://doi.org/10.1016/j.fbp.2019.02.001

\section{General rights}

Copyright and moral rights for the publications made accessible in the public portal are retained by the authors and/or other copyright owners and it is a condition of accessing publications that users recognise and abide by the legal requirements associated with these rights.

- Users may download and print one copy of any publication from the public portal for the purpose of private study or research.

- You may not further distribute the material or use it for any profit-making activity or commercial gain

- You may freely distribute the URL identifying the publication in the public portal 


\section{Accepted Manuscript}

Title: Optimal Operation of Parallel Dead-End Filters in a Continuous Bio-Based Process

Author: F.D. Bähner P.A. Santacoloma J.K. Huusom

PII: $\quad$ S0960-3085(18)30558-3

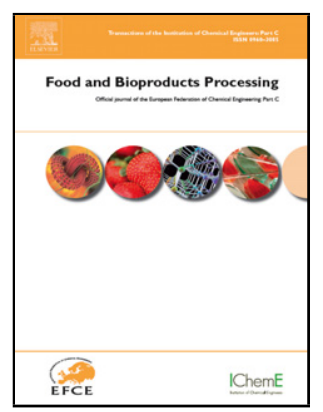

DOI: $\quad$ https://doi.org/doi:10.1016/j.fbp.2019.02.001

Reference: $\quad$ FBP 1040

To appear in: $\quad$ Food and Bioproducts Processing

Received date: $\quad 13$ August 2018

Revised date: $\quad 17$ January 2019

Accepted date: $\quad 8$ February 2019

Please cite this article as: F.D. Bähner, P.A. Santacoloma, J.K. Huusom, Optimal Operation of Parallel Dead-End Filters in a Continuous BioBased Process, <![CDATA[Food and Bioproducts Processing]]> (2019), https://doi.org/10.1016/j.fbp.2019.02.001

This is a PDF file of an unedited manuscript that has been accepted for publication. As a service to our customers we are providing this early version of the manuscript. The manuscript will undergo copyediting, typesetting, and review of the resulting proof before it is published in its final form. Please note that during the production process errors may be discovered which could affect the content, and all legal disclaimers that apply to the journal pertain. 
- Process-oriented analysis of the operation of parallel dead-end leaf filters

- Dynamics (non-linear batch process) especially of parallel filters are complex

- Application of plantwide control concepts to a full-scale industrial (bio-based) process 


\title{
Optimal Operation of Parallel Dead-End Filters in a Continuous Bio-Based Process
}

\author{
F.D. Bähnera , P.A. Santacoloma ${ }^{\mathrm{b}}$, J.K. Huusoma,* \\ ${ }^{a}$ Process and Systems Engineering Center (PROSYS), Department of Chemical and Biochemical Engineering, Technical University of \\ Denmark, 2800 Lyngby, Denmark \\ ${ }^{b}$ CP Kelco ApS, Ved Banen 16, 4623 Lille Skensved, Denmark
}

\begin{abstract}
Dead-end cake filtration as one of the first unit operations deployed in biochemical production plants has been subject of academic investigation for many decades. The recurrent discrete reinitialisation events are challenging from a process control point of view, especially in a continuous downstream line. The complexity that arises when multiple units are operated in parallel seems to have received little attention. This work aims at illustrating this complexity and delineates the arising plantwide control problem. Guidelines for optimised operation are derived from general process understanding and at hand of an industrial case study. The need for a predictive model to solve multiple scheduling problems is identified, and a mathematical model based on conventional filtration theory is derived. Due to raw material variability and operational uncertainties, the predictions are found to be too imprecise for deployment. This is expected to be representative of many bio-based processes, where manual scheduling needs to be integrated effectively into plantwide control structures.
\end{abstract}

Keywords: Dead-end Filtration, Full-Scale Process, Downstream Line, Biological Raw Material, Plantwide Control, Industrial Case Study

\section{Introduction}

Dead-end cake filtration is often one of the fundamental steps in the purification section of a biochemical production plant. As a workhorse for the separation of demanding slurries it must be regarded an established technology that, despite of trends such as continuous cross-flow and rotary-disk membrane technology, is far from being outdated. Especially due to the versatile applicability and robust separation properties, both of which have been demonstrated over many decades, it will retain its industrial significance for the time being. One frequently encounters filters which are operated manually, meaning that cake removal and reinitialisation, but also flow rate set point generation, need to be carried out recurrently by an operator. Automatically discharging filters are an answer at least to the labour-intensive reinitialisation. Their economic success was shown already in the 1970s (Rushton et al. (1996)). Manually operated filters on the other hand bear the advantages of allowing easy visual inspection of septum integrity, filter cake structures, and washing quality, and might thus not be replaced in the foreseeable future in some cases - not lastly due to large capital ${ }_{50}$ expenditure.

Operating parallel dead-end filters is non-trivial due to complex inter-filter dynamics, posing a scheduling problem. This work delineates the particular operational challenges that arise when parallel dead-end filters are utilised for the separation of compressible slurries in a continuous downstream line. The set of challenges is generalisable to many biochemical downstream lines, and they have been confirmed at hand of an industrial case study. The arising plantwide control problem especially for throughput and inventory regulation is analysed in detail, and the role of operators as central elements in the control structure design is examined.

The structure of the article is as follows: section 2 gives an overview of the process on a physical level, and delineates operational issues that arise when parallel filters are operated within a continuous regime. Thereafter, section 3 contains the findings from an industrial case study where a filtration unit and its role in a downstream line have been investigated. This includes an analysis of process data and a number of plant trials. Finally, in section 4 the control structures of downstream lines and particularly the case study plant are investigated in the light of the arising challenges. From this, it is concluded that a predictive filtration model would be most effective for augmenting decisions taken by the operators. Predictability is studied in section 5. where conventional filtration theory is reviewed with respect to its aptitude for generating on-line predictions, but falls short of the required precision.

\footnotetext{
* Corresponding author

Email address: jkh@kt.dtu.dk (J.K. Huusom)
} 


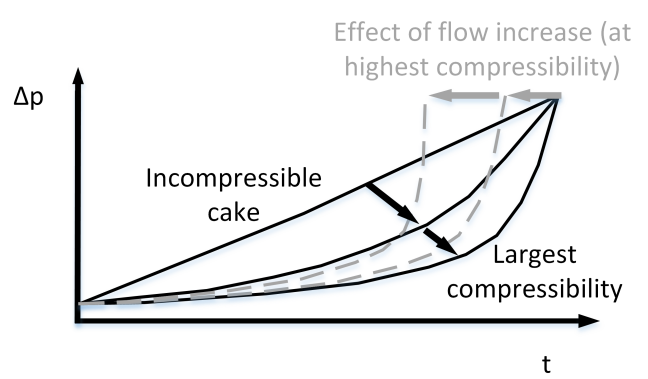

Figure 1: Schematic depiction of pressure cycle profiles at different compressibility and flow regimes over time.

\section{General Process System Description}

The following section starts with a delineation of the separation mechanism of a single filter. Thereafter, the description is extended to a scenario featuring multiple units with surrounding buffer tanks, similar to e.g. carousel chromatography (Jungbauer (2013)).

\subsection{Dead-end Cake Filtration Basics}

In a cake filtration processes, a slurry is pumped through a solid material-matrix such that particles above a certain particle size accumulate on the upstream side of the septum (or membrane). The septum itself is often a solid or woven semi-permeable material. As the cake grows thicker, an increasing pressure difference needs to be applied in order to maintain a steady flow of filtrate. A filtration cycle comes to an end when the maximum operating pressure of pump or membrane is reached. At this point, old filter cake needs to be removed. It takes a certain period of time to clean and reinitialise a filter, and no filtrate can be processed on the respective unit during this period. These discrete events are an inconvenience especially if the filters are part of a continuous downstream line. The overall operational time of a filter depends on the filter cycle length $t_{f}$ and the time it takes to clean and reinitialise a filter $t_{c l}$. The equipment availability

$$
\eta=\frac{t_{f}}{t_{f}+t_{c l}} \quad, \quad t_{f}=f(Q(t), \mathcal{Z}(t))
$$

represents the fraction of time a filter is actually ${ }^{100}$ processing filtrate. $\mathcal{Z}$ indicates disturbances such as slurry and precoat properties which, just as the operator steered flow rate $Q$, may change over time. They are decisive in defining the durations of the filter cycles. As $t_{f}$ is expected to vary significantly, so does $\eta$. An exemplary relationship between flow rate magnitude and equipment availability, which ultimately defines true filtrate throughput, is depicted in figure 2

\subsubsection{Cake Growth \& Filter Aids}

In practice, the septum is often coated with a layer of inert, rigid particles to increase the initial separation

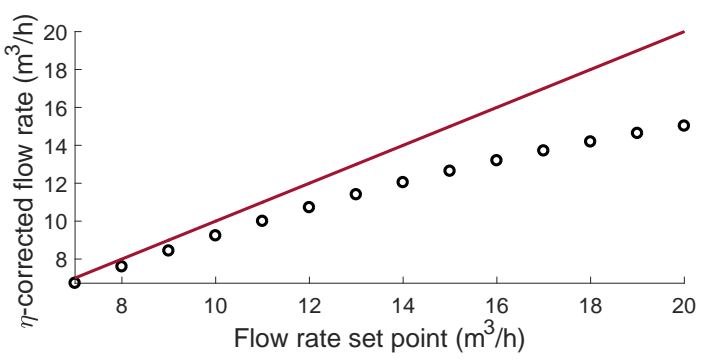

Figure 2: Representative, model-based calculation of the disparity between set point and time-averaged flow rate due to inactive times during re-initialisation.

properties. They may also be fed over time as filter aid, leading to a cake structure that improves filtration properties and longevity of the filter cycles. Smaller impurities contained in the slurry gradually adhere to walls and cavities while passing through these channels and are thus retained in the filter cake itself. Therefore, mechanisms of surface and depth filtration ordinarily coexist if the slurry contains solids of differing particle sizes. Blocking of channels has a negative effect on the permeability and accordingly lowers filtration capacity with time. Beyond cake height, characteristics such as particle shape and morphology, surface charge, and $\mathrm{pH}$ influence the pressure gradient throughout the bed (Tarleton \& Willmer (1997)).

\subsubsection{Cake Compressibility and Blinding}

Material-deformation under stress gives the pressure cycles their power-law like curvature. This is depicted schematically in figure 1 (for a real-life example find figure (4). A further complication arises as cakes can 'blind'; that is channels and pores collapse if a sudden pressure shock is applied. In everyday-practice, this can occur if a flow rate is increased at a late point in a filtration cycle. This decreases the volumetric capacity of that filter cycle and is thus undesirable. Lastly, in addition to the reduction in capacity, a large pressure step-up can cause cracks or detach accumulated small particles, upsetting product quality.

\subsection{Extension to Systems of Parallel Filters}

The equipment availability factor is generally considered in the design process, leading to the right number of appropriately dimensioned units (see e.g. Casas-Orozco et al. (2015)). However, in the design process, little regard is paid to the challenges that arise during everyday operation, where slurry conditions change drastically. Beyond this, due to cross-scale issues (Tarleton \& Hancock (1997)), it can be expected that the laboratory-determined capacity differs from the real capacities; a problem normally solved by over-dimensioning surface areas and pumps. Therefore, it should be considered good practice to determine the true 
capacity of the full-scale equipment after it has been commissioned, as line capacity will ultimately be pushed during demand peaks.

Operating parallel filers enables the employees to mitigate the discontinuity-effect of a filter cleaning by simultaneously increasing the flow rates on other active units. Note however that, if cake compressibility is an issue, this may lead to cake blinding. It is therefore a practical, yet sub-optimal solution in terms of equipment capacity utilisation. As buffer tank over- and underfill need to be avoided, inactivity of a filter can lead to forced flow rate adjustments on other filters. Alternatively, flow rate changes on the unit operations upstream or downstream can become necessary, but might be inconvenient if the unit is sensitive to set point changes.

Therefore, it is in general desirable that filter inactivities do not overlap, which amplifies the effect of the discontinuities. This is of especially high importance if the filters are reinitialised manually, as human labour is usually a limiting factor due to high personnel costs. As a consequence, if no operator is free, a filter remains inactive for longer than the minimum reinitialisation time. This not only upsets buffer tank levels unnecessarily, it also reduces line capacity due to the decrease in equipment availability.

\subsection{Classification of Problems}

Most of the challenges delineated in the above are interesting from a control engineering perspective. They are summarised in the following:

I States are strictly transient (batch).

II Parallel units are in need of active scheduling.

III Strong non-linearities (compressibility of materials).

IV Buffer tank levels are inherently noisy $\rightarrow$ complex inventory control task.

V Usually, operators regulate the process, thus human factors play a role (manual control).

VI Cake blinding as a cause of flow increases (actuation) moves the capacity constraint.

VII Most relevant process parameters (concentration of filtered impurities, cake properties) cannot be measured effectively for control purposes.

To the best of the authors' knowledge, while pressure filtration has received significant attention regarding the design of equipment and characterisation of slurries, operational aspects are largely neglected in the academic community. Especially in the plantwide context of a continuous downstream line, the above challenges make it an interesting problem that is quite representative of the situation in the broader bioindustry. Filters furthermore allow the relatively facile implementation of plant trials at full scale without upsetting product quality, as the separation by means of size-exclusion is a robust mechanism. In the following section, it is to be inquired at hand of a case study, to which extent the challenges derived from process fundamentals in the above are a concrete issue in everyday production.

\section{Pressure Filtration in Practice: A Case Study}

The filters under investigation are located within a pectin production line operated by $\mathrm{CP}$ Kelco ApS. Pectin, a gelling and thickening agent used predominantly in the food industry, is generally leached from citrus peels in acidic extractions. The pectin rich liquid is quite viscous, rendering solid-liquid separations difficult. CP Kelco agreed to the publication of process data related to the pressure filtration step, which is known to be a potential bottleneck. It is not always possible to determine whether slurry properties or suboptimal operation are the cause.

\subsection{The Pectin Extraction-Purification-Evaporation Line}

The pectin production line (up to the evaporation step) is used to delineate the complex task of controlling a biochemical downstream line. It is schematically depicted in figure 3 .

Pectin Extraction: At all times, a number of parallel batch extractions evolve on a planned schedule. The amount of peel in an extraction tank, $\mathrm{pH}$ as well as temperature constitute the most influential process parameters; extraction time is ultimately determined by batch schedule and plantwide liquid throughput. Conditions are tuned in batch-to-batch manner after a qualified first guess is made based on peel pre-processing results. If a sustained bottleneck arises in the downstream line, this disturbs the schedule, prolonging extractions and degrading pectin quality.

Re-Extraction and First Coarse Filtration: when an extraction is complete, the tank's content is fed to a number of continuous filters that remove most of the cellulose remnants. The tanks are emptied one-at-a-time - without interruptions, as cake integrity on these filters otherwise degrades. The filters are adjusted such that all upstream material is processed steadily, and the cake is fed to a second extraction (yield increase). A further coarse filtration in the recycle stream ultimately purges the (essentially entire) solid phase from the system. The extraction slurry is mixed with re-extraction filtrate before being fed to the coarse filters to lower viscosity. (Due to lower pectin concentration and quality, the re-extraction liquid is less viscous.) The process is operated optimally when the re-extraction broth lowers the viscosity of the overall process fluid to a filterable level.

Second Coarse Filtration: freed of the largest solid particles, the filtrate is fed to a system of further parallel 


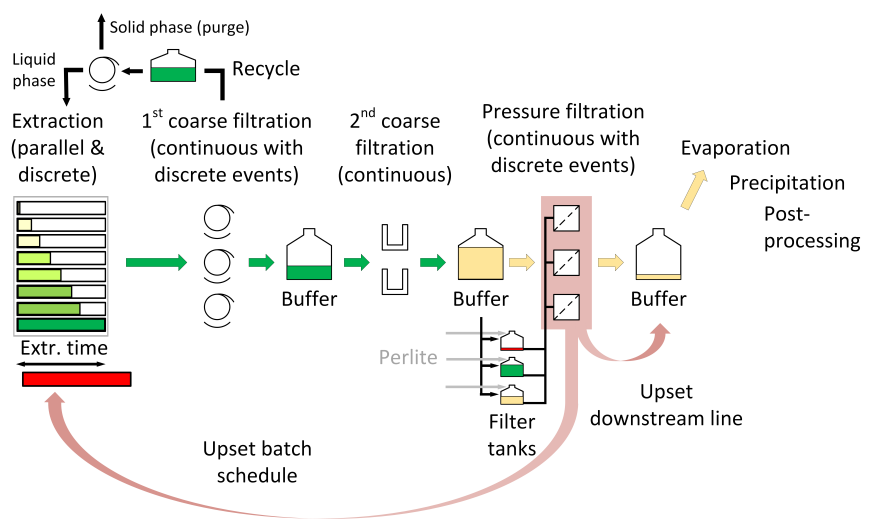

Figure 3: Extraction-purification sub-system: the pressure filters threaten to bottleneck the plant, a) due to faulty scheduling or b) in extreme cases if cycle times even are very short, not enough manpower is available - this upsets batch-schedule and evaporator.

solid-liquid separators, a sizeable surge tank is installed between the units. The flow rate on these machines can be adjusted flexibly, thus a level controller propagates the upstream throughput in the direction of flow.

Pressure Filtration: finally, in the micro-filtration step, vertical perlite-precoated dead-end leaf filters are used to remove smaller impurities. These units guarantee a reliable split, independent of fluid \& process conditions. A detailed description of this sub-system is given in section 3.2 and thus foregone at this point.

Evaporation: the pectin extract is concentrated to facilitate the precipitation. It is desirable to run the evaporator close to the point of sticking, which is non-trivial as stuck material may quickly burn, upsetting product clarity. This loss in quality cannot be recovered and has strong negative economic implications. Therefore, operators dislike adjusting set points if the conditions on the evaporator have been properly configured for a fluid with a certain viscosity and pectin concentration.

\subsection{Detailed Assessment of the Industrial Pressure Filtration Step}

A knowledge-base is built by conducting contextual interviews with the operators (Beyer \& Holtzblatt (2010) ) and observing operations. Furthermore, process data from five filters, taken over a month, have been analysed. All filters are of identical design, however, two of the units have smaller membrane areas. Due to the number of considered cycles (265), manual processing of the pressure profiles was not feasible and an algorithm has been developed to extract them from the time-series data.

\subsubsection{End-Time Scheduling: Challenges I - III}

It is confirmed that the first three challenges derived from process fundamentals in section 2.3 are indeed cause
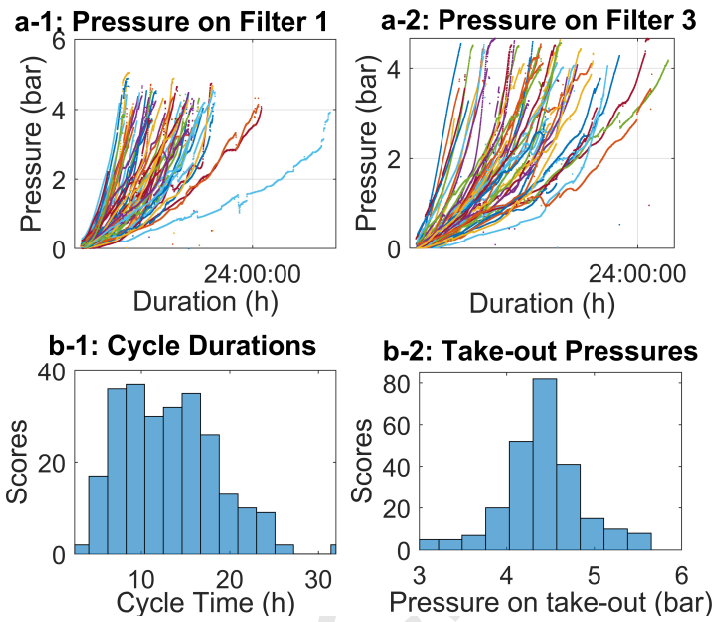

Figure 4: Pressure profiles for a small (a-1) and a large filter (a2 ). Histograms of filter cycle durations (b-1) and take-out pressures (b-2).

of some trouble. Operators try to align inactive periods such that they do not overlap by adjusting filtrate flow rates towards the later stage of filtrations. The filters are cleaned manually, meaning that after terminating a cycle, the filter vessel is opened, leafs and supporting rack exposed. Finally, the membranes are hosed down with water in order to remove the filter cake. Thereafter, the vessel is reinitialised, which includes running in precoat-recycle until all filter aid is evenly distributed on the membranes. Especially in low-wage countries, manually reinitialised filters are likely to retain their importance also in the future for large-scale pectin production (Casas-Orozco et al. (2015).)

Due to the amount of information (multiple filters) and the non-linearity of the profiles, anticipating the end of all filtrations is a complex task. This is furthermore complicated by the fact that cycle times vary drastically. The extent of these uncertainties can be estimated from figure 4 a-1, a-2, and b-1, from where it becomes evident that cycles may end anywhere between 5 and 30 hours. Cleaning on the other hand is a standardised procedure and usually takes between 45 and 60 minutes. Cycle duration largely depends on the volumetric capacities (filtrate volume per cycle before reinitialisation), which is shown in figure 5. A number of factors, i.e. fluid properties defined by product and raw material type (with substantial variability, Caroco et al. (2018)), but also operational uncertainties shape the capacities: while operators are advised to terminate filtrations only after passing the 4.5 bar threshold, in reality a distribution with a mean below 4.5 is seen (figure $4 \mathrm{~b}-2$ ). This may occur due to suboptimal scheduling, but can also be a cause of too little disposable filter aid (next section). Taking a filter out ahead of time (i.e. before membrane potential is reached) evidently has negative implications for capacity and is undesirable. Lastly, it became evident 


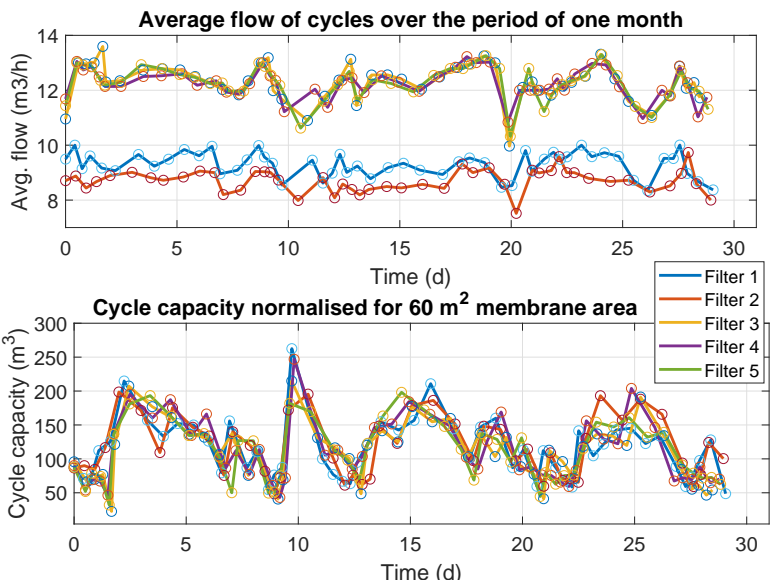

Figure 5: Average filter flow rates (scaled to mask plant capacity) and the according cycle capacities. A circle corresponds to cycle ending.

that operators tend to try to end the filter cycles in groups (consecutive). This is convenient for them, as they can hose down multiple racks but only have to equip the necessary safety equipment once. However, it exacerbates the scheduling task, as it induces a higher risk of inactivity overlaps and thus bottlenecking.

\subsubsection{Filter Aid Dosing: An unforeseen Complication}

Product quality is the paramount production objective, and the filter aid strategy (type, i.e. granularity of perlite) is chosen such that adequate clarity is assured: the finer the perlite grade, the better product clarity. Yet, finer grades of perlite are more ensive, and at the same time reduce volumetric capacity of the cycles. This decreases line throughput and increases overall perlite uptake due to the use of more material in the thus more frequently occurring reinitialisations. Therefore, a balance is carefully found based on quality feedback.

Generally, precoating is essential to assuring proper performance. Filter aid furthermore needs to be supplied throughout the filtrations as body feed; the grade can differ from that in the precoat layer. Each filter is equipped with an auxiliary tank that can hold up to 5 $\mathrm{m}^{3}$ of filter aid mix. The tanks need to be replenished before running dry in order to allow continuous feeding into the feed-stream of the filter vessels. The amount is set in a fixed ratio relative to filtrate flow rate. Refills ordinarily occur up to 3 or 4 times during a filter cycle, depending on its volumetric capacity (longer cycles need more filter aid tank refills). A complication lies in that a filter aid tank needs to run dry when a filtration ends, otherwise remaining content needs to be purged in order for the tank to be empty when the precoat mix is prepared. This is a simplification, but describes the problem to a large enough extent. Details can be found in Bähner et al. (2018). At this point, it suffices to understand that operators therefore cannot fill the filter aid tank to an arbitrarily high level in the last refill procedure. Due to the arising workload, it is not feasible to refill the tanks in incremental amounts, either. Thus, the absence of sufficient filter aid can ultimately lead to a situation where a filtration is ended before reaching the threshold pressure $(4.5$ bar $)$, with the negative capacity implications thereof.

\subsubsection{Inventory Control: Challenges $I V-V$}

The amount of liquid necessary for the preparation of filter aid \& precoat mixes is taken from the upstream pressure filtration buffer. This sudden draining of material is perceived as a quasi-discrete drop in the buffer tank level. Furthermore, as expected, inactivity of a filter leads to a accumulation or depletion of material in the respective buffer tanks which are therefore usually in transient state. As Zheng \& Mahajanam (1999) point out, processes exist where the installation of large buffer tanks is economically optimal, as the otherwise more complex control task has harsher economic implications this seems to be the case. Still, operators sometimes have to change filter flow rate set points to avoid over- or underfill (manual level control). Looking at figure 6, it can be seen that the buffer tank levels are unsteady and run into extreme peaks; sometimes concurrently, therefore likely due to an upstream bottleneck such as in o day 18. The downstream buffer can also deplete due low cycle capacities, e.g. figure 5 -bottom, days 5 to 9 . Note also, how in this period, the flow rate set points in figure 5-top need to be increased to account for the dwindling equipment availability. The downstream buffer is therefore traditionally kept at a high fill-level in order to be able to overcome a temporary shortage of filtrate. This in turn implies a risk of overfill, and might thus lead to unnecessary filtrate flow decreases. As mentioned earlier, the operators prefer not to change the flow rate on the evaporator if it functions smoothly, thus they likely shift material around in the filter buffer tanks until the mass balance is no longer closable in this way, and only then adjust the evaporator. To aid the operators who might be occupied with e.g. a reinitialisation, a safety override has been implemented that shifts material from the upstream buffer to the pre-second-filtration tank by automatically lowering the inflow if the upstream tank level surpasses a threshold.

\subsubsection{Compressibility and Cake Properties: Challenges $V I-V I I$}

Dynamic effects such as particle rearrangement may vigorously influence the cake structure, as pointed out e.g. by Tarleton (1998). Therefore, the quantification of compression effects is important in order to specify operational guidelines that maximise capacity. Due to changing fluid properties / cross-scale issues, laboratory experiments are not conveyable to full-scale. Therefore, actual process data was taken into account. There are 

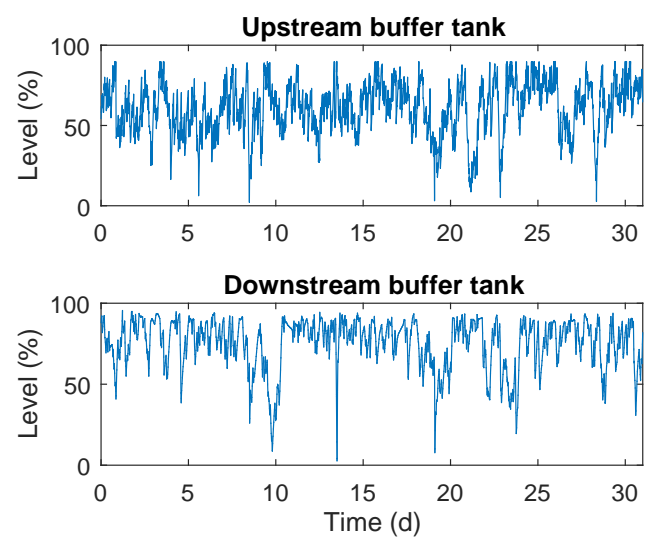

Figure 6: Historical process data: Buffer tank level evolution over time. High level in downstream tank leaves little room to accommodate oscillations but can temporarily supply downstream units in case of filtrate shortage.

not usually extensive periods of stasis (figure 5-bottom), which renders statistical analyses difficult, as trends might be hidden behind transient behaviour. Still, in the following it is intended to monitor capacities of cycles that have been clustered into a population with likely compression effects, and one that is certainly unaffected in a longitudinal study.

The cumulative flow of filtrate that can be processed with one filtration cycle depends, among other factors, on the final pressure at take-out. With this in mind, and to reduce the effect of operational uncertainty, a common base is created by comparing cycles at certain pressure thresholds. The filtrate throughput is calculated using trapezoidal integration on a minute basis, which is sufficiently accurate. In the evaluation algorithm, the time at which a certain pressure is reached is identified for each cycle. Thresholds have been set to 1,2,3 and 4 bar (4.5 bar would imply that a large fraction of the cycles cannot be included due to earlier take-out, see figure 4 b-2). The cumulative filtrate for each cycle at each pressure threshold, namely between $t_{p_{0}}=0$ and $t_{p_{i}}=t\left(p=p_{i}\right)$ is then calculated:

$$
V_{i}=\int_{t_{p_{0}}}^{t_{p_{i}}} Q(t) d t, \quad i=1, \ldots, 4 .
$$

The average flow rates up to a threshold are calculated as

$$
\bar{Q}_{i}=V_{i} /\left(t_{p_{i}}-t_{p_{0}}\right) .
$$

Controlled plant trials were carried out to investigate the effect of compression. As two of the filters are equipped with smaller surface areas, it was convenient to utilise them, minimising the implications on the daily routine. However, the operating crews believe that, despite of the equal sizing and machinery, there is a certain difference in performance. This is causing them to run the filters at disparate flow rates. A brief analysis of the data suggests that they likely function equally well (table 11 less flow
Table 1: Statistics of the two smaller filters in everyday production. Difference in average durations is likely to be induced by disparate flow rates, not equipment differences.

\begin{tabular}{ccc}
\hline & Filter 1 & Filter 2 \\
\hline Avg. flow rate $(\mathrm{m} 3 / \mathrm{h})$ & 9.3 & 8.7 \\
Avg. cycle duration $(\mathrm{h})$ & 12.6 & 14.1 \\
Avg. total filtrate $(\mathrm{m} 3)$ & 92 & 94.5 \\
Nr. of cycles per month & 53 & 48 \\
\hline
\end{tabular}

Table 2: Capacity validation of small filters, 20 cycles for each unit: Filtrate throughput $\left(\mathrm{m}^{3}\right)$ up to pressure threshold. Notation: Mean $\mu$, standard deviation $\sigma$.

\begin{tabular}{r|cc|cl}
\hline $\begin{array}{c}\text { Threshold } \\
\text { (bar) }\end{array}$ & 1 & 2 & 3 & 4 \\
\hline Filter 1: $\mu$ & $\begin{array}{c}73.3 \\
(33.5)\end{array}$ & $\begin{array}{c}119 \\
(43.1)\end{array}$ & $\begin{array}{c}148.9 \\
(49.4)\end{array}$ & $\begin{array}{l}158.6 \\
(51.2)\end{array}$ \\
\cline { 2 - 5 } Filter 2: $\mu$ & 75 & 117.5 & 145.9 & 158.1 \\
$\sigma$ & $(34.2)$ & $(39.5)$ & $(45.9)$ & $(48.2)$ \\
\hline
\end{tabular}

leading to longer cycles and higher capacity). Therefore, an initial plant trial is carried out to in order to investigate this further.

\section{Trial I: Capacity Verification}

It was decided to run the filters at equal constant rates for three weeks, accounting to a total of 20 cycles per filter. The results are depicted in table 2 and a notable degree of operational uncertainty, causing large natural standard deviations, must be acknowledged. However, looking at the mean values and having the knowledge that both filters have identical equipment specifications, it has been concluded that they perform comparably.

Trial II: Effect of Flow Rate Magnitude on Cycle Capacity

It is believed that filtrations which are run at high flow rates generally yield less filtrate per filtration cycle due to an overall higher pressure regime, causing faster cake compression. Therefore, the filters are run at disparate, constant flow rates in a second trial, isolating the effects of flow rate magnitude on filtration cycle capacity. The results are presented in figure 17, where the final amount of filtrate that was processed at lower flow rates is seen to lie ca. $10 \%$ above the other filter at the 4 bar threshold. A critical remark: the number of cycles run on the filters is quite different due to the lower / higher cycle durations: $9\left(\mathrm{~m}^{3} / \mathrm{h}\right) / 5\left(\mathrm{~m}^{3} / \mathrm{h}\right)=1.8$. In addition, there is a reduction in equipment availability due to higher number of reinitialisations, and also here, substantial process noise is experienced. Still, as the filter run at lower flow rates performs consistently better at all pressure thresholds, it should be concluded with some certainty that there is a negative implication when running at unnecessarily high flow rates, which corresponds to what process understanding indicates. 


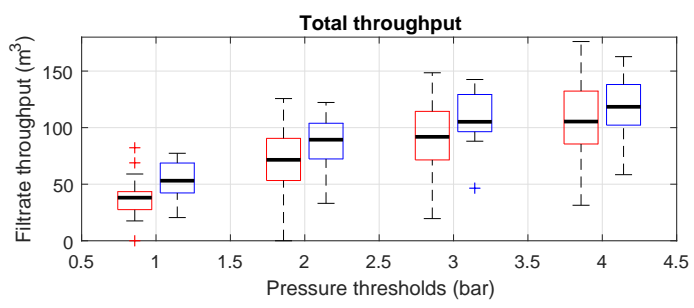

Figure 7: Performances at disparate, constant flow rates (Red boxes: $9 m^{3} / h$. Blue boxes: $\left.5 m^{3} / h\right)$. Large standard deviations due to lack of stasis in data-set.
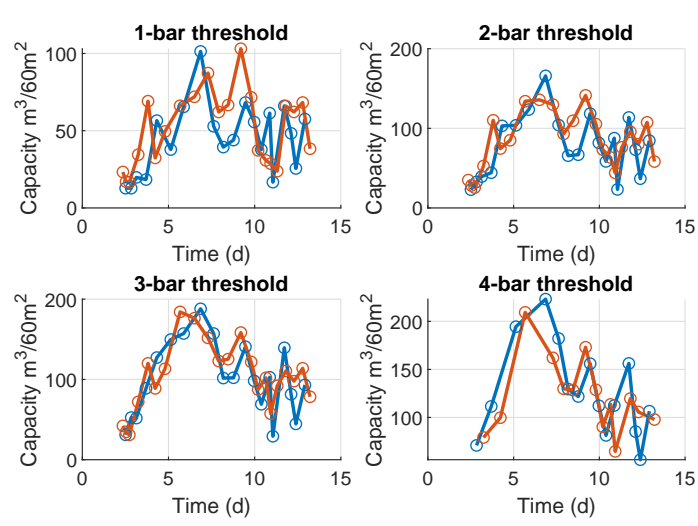

Figure 8: Performances for initially equal constant flow rates (Blue: No Increases. Red: Increase of $1 \mathrm{~m}^{3} / \mathrm{h}$ at 2 and 3 bar). Volumetric capacity of cycles during trial changes drastically with more than $150 \mathrm{~m}^{3} / 60 \mathrm{~m}^{2}$ membrane.

\section{Trial III: Effect of Flow Rate Increases on Cycle} Capacity

In the last plant trial, operators were asked to restrict one of the units to constant rates, whereas on the second unit a distinct flow rate increase of $1 \mathrm{~m}^{3} / \mathrm{h}$ was to be implemented upon reaching 2 and 3 bar. Overall, the operators struggled with the task due to the general workload, which is why they missed the specified point in time for some cycles, but a number of appropriate samples were generated. The trial is complicated by the fact that fluid properties during this period were changing drastically, as can be seen in figure 8. Tracking the evolution of the means of the populations indicates that cake blinding has a significant negative effect. Note also that parts of the red line lie significantly above the blue at the first three thresholds, yet not so thereafter. Still, due to the above-mentioned inhibitors, no statistically significant conclusions can be drawn from trial III.

Assessment of Cake Compressibility based on Historical Data

Trying to single out the effect of cake blinding, the historical data set is taken into account. In a comparative study, the easiest measure is a separation into a group of cycles during which the flow has been ${ }_{400}$
Table 3: Assessment of compressibility losses at hand of historical data. Filtrate throughput $\left(\mathrm{m}^{3}\right)$ up to pressure threshold, samples grouped into cycles with / without increases. Notation: Mean $\mu$, standard deviation $\sigma$.

\begin{tabular}{ccccl}
$\begin{array}{c}\text { Threshold } \\
\text { (bar) }\end{array}$ & 1 & 2 & 3 & 4 \\
\hline No increases: $\mu$ & $\begin{array}{c}56.5 \\
(26.3)\end{array}$ & $\begin{array}{c}90.7 \\
(42.5)\end{array}$ & $\begin{array}{c}113.8 \\
(51)\end{array}$ & $\begin{array}{l}128.5 \\
(52.3)\end{array}$ \\
\cline { 2 - 5 } & $\begin{array}{c}(26.5 \\
\text { Increases: } \mu\end{array}$ & $\begin{array}{c}96.4 \\
(35)\end{array}$ & $\begin{array}{c}114.5 \\
(42.8)\end{array}$ & $\begin{array}{l}121.1 \\
(50.3)\end{array}$ \\
$\sigma$ & $(27.1)$ & $(35)$ & 193 & 135 \\
Cycles w/o incr. & 215 & 203 & 66 & 89 \\
\hline Cycles w. increase & 50 & 61 & 66
\end{tabular}

increased, and one where that is strictly not the case. However, if done so, even if a flow rate is elevated for a negligibly short period only, the cycle will be classified as compression-affected. It is understood that particle rearrangement takes at least some time. Thus, a more refined criterion was introduced, utilising a decision variable at each threshold pressure:

$$
C\left(p_{i}\right)=\sum_{i} \delta Q_{i}\left(t_{\text {final }_{\text {Segment }}}-t\left(\delta Q_{i}\right)\right)
$$

Here, $\delta Q_{i}$ denotes a change in flow rate and $t\left(\delta Q_{i}\right)$ the corresponding time-of-change. Verbalised, cycles are (irreversibly) clustered based on the amount of filtrate that has been processed at an elevated flow rate. Cycles with questionable compression effects are excluded, allowing a comparison between cycles with significant compression effects and those clearly unaffected. Therefore, the total number of cycles in table 3 is decreasing (it can also be due to a cycle ending before passing a threshold pressure).

Ultimately, the mean of total processed filtrate suggests that cake compression has a negative effect on capacity, accounting to roughly $6 \%$ of the total (table 3). Furthermore it appears that operators are more likely to increase flows on cycles that run well in the early stages. These events do not constitute scheduling actions, which occur only toward the later stages of a cycle and are expected to be more costly in terms of capacity. Notably, filtrations with outstanding performance are exclusively associated with the compressibility-unaffected population. This is supported by experiences from the production site, where operators believe to have observed cycles ending suddenly upon late-stage flow rate increases. Overall, it seems that cake compressibility induced losses are likely significant with ca. 5 to $10 \%$ of cycle capacity.

\section{Discussion: Plantwide and Local Control Strategy}

In the following, it is to be discussed whether there are evident shortcomings in CP Kelco's operational 
paradigm, and furthermore if technological tools can be envisioned that may aid in optimising plantwide operations. Due to the absence of a high-fidelity plantwide model, batch-operated extraction tanks and filters, as well as the overall restriction to the filtration-evaporation section, successful complete application of a process-oriented or hybrid (Vasudevan \& Rangaiah (2012)) plantwide control structure synthesis method (Luyben et al. (1997); Luyben (2012); Skogestad (2004, 2012); Murthy Konda et al. (2005)) was deemed problematic. Therefore, the most relevant process-oriented concepts have been extracted and used to decompose essentials of the plantwide control problem into tangible sub-task. In general, the application of plantwide control to bio-based production plants (e.g. Van Dijk et al. (2009)), disregarding the highly automated, continuous production of bio-fuels (e.g. Ochoa et al. (2010); Patle et al. (2014)), is not frequently documented. It is likely that a cost-drive push for integration and intensification as well as the Quality by Design framework will see focus being put on conclusive plantwide (arguably, i.e. quality) control strategies (Rathore \& Kapoor (2016); Wu et al. (2007)) in bio-based production facilities.

Rijnsdorp (1991) and most ensuing plantwide control researchers suggest that economic performance needs to be assessed in the light of the current market situation. Specifically, in a sellers market (maximum throughput) scenario, capacity needs to be maximised as shortage cost is likely to exceed the cost of utilities, which is equivalent to de-bottlenecking the plant if there is an obvious local capacity constraint. In the economically less desirable but often unavoidable scenario of a buyers market (nominal operation), engineering and operational focus should rather be put on minimising costs. A convenient consequence of producing below maximum capacity is that temporary throughput bottlenecks can be compensated in the future without negative implications, so long as no extra costs arise (Skogestad (2002)).

Applying these concepts to the situation at CP Kelco, it is found that the pressure filters are likely to be both: capacity limiting constraint in the maximum throughput case, but also temporary bottleneck due to possible maloperation (e.g. scheduling errors) in a nominal throughput scenario. A temporary bottleneck, if it persists long enough, eventually propagates upstream and delays the extraction schedule. This leads to quality degradation, therefore temporary bottlenecks have negative economic implications in a nominal throughput filter cycle capacity' depends on how operational degrees-of-freedom are used (cake blinding as a cause of flow actuation), de-bottlenecking the filtration area by means of improved operation is especially interesting for the maximum throughput case.

\subsection{Operational Objectives}

The process is predominantly sequential, which, in combination with large buffer capacities, is expected to facilitate the plantwide control task, at the cost of higher operational expense (Stephanopoulos (1983); Price \& Georgakis (1993)). Still, the global objectives (maximise capacity vs. minimise costs and risks) need to be translated into differentiated local objectives. In both cases (max. and nom. throughput), it is optimal to operate the filters such that the take-out pressure is maximised, as this increases capacity and reduces perlite usage. (Only if an end-time collision is unavoidable, it might be preferable to reinitialise the filter at a lower pressure to avoid coinciding inactivity.)

One of the largest challenges is that the constraint (volumetric filtrate capacity per cycle, figure 5) moves almost constantly, depending on raw material and product. This ultimately translates into cycle time $t_{f}=V_{f} / Q$ and filter cleanings per $8 \mathrm{~h}$ shift: $N_{c l}=8 h \times N_{u} /\left(t_{f}+t_{c l}\right)$, where $N_{u}$ is the number of parallel units.

\subsubsection{Maximum Throughput}

In general, for the aggregate production unit (parallel filters) two objectives could be identified:

- Operate the units such that the number of total manual filter cleanings is minimised with maximal spacing between the end-times.

- Use manpower efficiently by maximising the amount of cleaned filters per operator and shift.

This is motivated by the fact that human labour ultimately is the throughput limiting factor (operators cannot keep up the cleaning schedule if the frequency is too high). The operator load can be expressed as

$$
\xi_{\text {operator }}=\frac{N_{c l} t_{c l}}{t_{\text {shift }}} .
$$

Idealising, the filtration area would at all times be run such that operators do not experience any idle times between consecutive filter cleanings (figure 9a) - paying idle workers is uneconomical. In reality, some distance in the spacing is required to give the operators enough time to engage in the necessary tasks for optimal capacity usage on a single machine (section 4.1.3). Setting flow rates appropriately requires conscious planning, which is likely to be flawed if the workload is too high. Furthermore, as Rijnsdorp (1981) points out, a long-term operational strategy needs to be found that allows for an adequate level of job satisfaction. And finally, as uncertainty in the end-times is expected, some spacing between the filters can be desirable to minimise the risk of inactivity overlaps with the negative capacity implications thereof. Any scheduling uncertainty above that is, strictly speaking, sub-optimal if throughput is to 


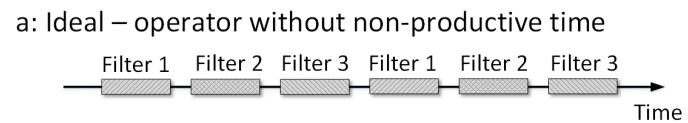

b: Uncertainty leads to non-productive time

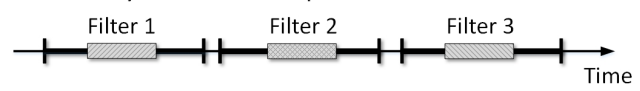

Figure 9: Ideal and real filter reinitialisation events (grey bars) during an operator shift for a scenario where filters are the dominant bottleneck.

be maximised and filters are the limiting step (figure $9 \mathrm{~b}$ ). How closely the cleaning frequency / capacity constraint can be tracked depends on the one hand on the extent of the operational uncertainties, but also on how steady the fluid properties are. It is not feasible to keep up with drastically changing constraints, thus throughput can be maximised more efficiently in periods with few material and product changeovers.

If filter cycle capacity is to be truly maximised, a constant rate focussed operational regime at maximum, equal spacing of end times is necessary, as it avoids capacity losses due to cake compression. This requires consciously shifting the process inherent variance from flow rates to buffer tank levels. Until now, the downstream buffer tank level set point has been at around $90 \%$ (figure 6), making it a material buffer. This should be lowered to a value between 50 and $60 \%$ - in order to allow the oscillations that are necessary to accommodate the variance, rather than providing extra material. Overall, the gain from constant rate operation might not justify the increase in operational complexity.

\subsubsection{Nominal Throughput}

In this case, some idle time for the operators is likely unavoidable if the shifts are staffed for the 'worst case scenario' cycle durations. It should be assured that the product concentration is high even at low throughput by supplying sufficient raw material to the extractions, as unnecessary water has to be removed. This will likely reduce cycle durations due to a viscosity increase, but not necessarily lead to full-utilisation (equation (5)) due to the lower flow rates. If discontinuities therefore arise less frequently, this could allow the buffer tanks to be kept at lower inventory levels, making product changeovers less costly due to reduced residence times. Overall, it would be desirable if operators could engage in further value-creating activities (at other process stages), as the objectives 'maximum spacing between end times' and 'effective operator utilisation' work against each other.

An unforeseen bottleneck in a nominal throughput scenario can arise if impurity load or viscosity are too high for the filters. This can happen if too much unquantified peel is fed to the extraction tanks after a peel changeover. As it is undesirable to have low product concentrations, it could be feasible to optimise the peel amount while extracting on a 'slower' extraction schedule, meaning that the tanks are started after longer intervals. When the peel amount is optimised, the starting interval and therefore liquid flow rate through 550 the plant could be increased, thereby avoiding bottlenecks with the negative implications for quality. This is, however, a rather complicated procedure, and modern peel pre-processing tools might be a simpler solution.

\subsubsection{Optimal Filtration Conditions}

In order to ideally exploit available capacity on a single filter, it is necessary to

- Initialise a filtration cycle with the appropriate flow rate, needed to

- Restrict / minimise late flow rate increases.

- Prepare the precoat layer properly.

- Set the correct filter-aid dosage and grade.

- Operate filtrations until maximal pressure is reached.

Adjusting any of the above to an optimal value in a strongly disturbed industrial context is, however, not trivial. Especially adjusting perlite grade and ratio requires careful end-point quality feedback, which is a slow procedure. According to Wang (2006), the optimal body feed can be identified at hand of the curvature of the pressure profiles. In general, filter aid should be dosed such that cycle durations are maximised - roughly to the point where an increase does not enhance performance further. If conditions change persistently, it is unlikely that the optimum feeding strategy can always be found, thus a robust solution that guarantees sufficient clarity should be chosen. Controlled long-term plant trials and careful data evaluation such as presented in section 3.2.4 are advised. Sustainable flow rate set points

$$
Q_{S P}=\frac{Q_{\text {plant }}}{N_{u}} \frac{1}{\eta}
$$

need to be generated if true constant rate operation is desired. Here $N_{u}$ denotes the number of parallel units which, for understandability reasons, are assumed to be equal-sized. The disparity between sustainable and current flow rates becomes more pressing in the case of short cycles with large flows. Negligence of $\eta$ may thus lead to a significant drift in the naturally oscillating buffer tank levels.

\subsection{Location of the Throughput Manipulator}

Skogestad (2012) strongly suggests locating the throughput manipulator (TPM) in the vicinity of the bottleneck. This allows carefully driving the process as 


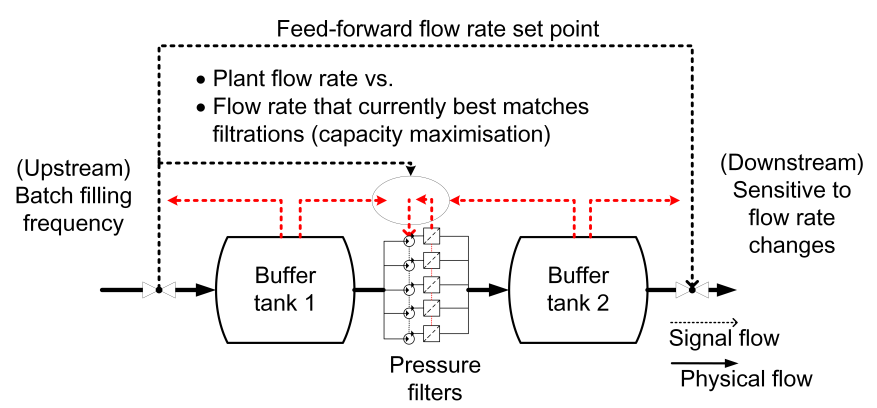

Figure 10: Under-actuation of the 2-buffer system during long periods. Flow rates that optimise filter cycle capacities can be in disparity with the plantwide flow rate.

close as possible to the most pressing constraint; a property that is deemed important especially if switching from nominal to maximal throughput. The reality at $\mathrm{CP}$ Kelco is that this constraint is usually changing (see cycle capacities in figure 5), and hardly predictable.

Manipulation of either filter flow rates or evaporator throughput to set plantwide throughput is impractical, as the batch extraction schedule would be upset, jeopardising quality - the situation for a fermentation plant is believed to be identical. This inertia in the schedule needs to be acknowledged. Thus, liquid throughput should be set consciously at the front-end of the process by selecting the batch filling interval, not via an internal TPM - with the negative implications of a slower system response (Price et al. (1994)). As central operators can steer the entire flow scheme manually, they try to incorporate an experience-based feed-forward aspect, as indicated in figure 10. As a consequence of this, the filtration-buffer tank system is regularly under-actuated: the cumulative filter flow rate (one ${ }_{650}$ manipulated variable) is adjusted to avoid over- and underfill in two buffer tanks, reacting to the more pressing variable. Only in cases of infeasibility are upstream or evaporator throughput adjusted to close the mass balance

As the front-end of the plant is fed with a liquid as well as a solid phase, there are two mass balances to be closed. Yet, most solids are purged in the 1st stage filtration step, thus the solid phase can be largely ignored in the later stages, also in the pressure filtration. However, the amount of impurities as well as product concentration are largely defined by the amount of peel in the extractions and shape filter cycle capacities. Thus the peel load to the tanks can be used to carefully drive the process to a feasible region after a peel changeover. Again, energy is wasted in the evaporation step if the product concentration is too low, thus a steep concentration ramp-up and a qualified first guess are important.

\subsection{Inventory Control}

The dynamic behaviour arising in and between the buffer tanks is complex, and may force the operators to act in a manner that is suboptimal in a capacity utilisation sense (e.g. calling for a sudden flow increase, or shifting cycle end-points in an undesirable direction). These complex procedures are prone to human error, especially in stressful situations (Mannan (2012)). In general, as the evaporator flow rate is often set in a feed-forward manner depending on the batch interval by the central operating crew, the inventory control structure cannot be seen as locally consistent Aske \& Skogestad (2009)). This motivates the search for an improvement utilising automatic level control - under the assumption of an upstream throughput manipulator to propagate the mass balance downstream. However, the discontinuities lead to a significant amount of process-inherent variance. Due to the possible negative effect on capacity, it is desirable to capture this in the buffer tank levels rather than transmit it to the filter flow rates. The extent of the process-inherent (that is without material- or operator induced) uncertainties can be taken from figure 11, where the 'best-case' scenario of constant flow rates and exact take out pressures has been simulated, setting the flow rates according to equation (6) and respecting all regular discrete events. This renders the implementation of a 'simple' inventory controller such as e.g. detuned proportional controller (Faanes \& Skogestad (2003)) or the optimal averaging controller (McDonald et al. (1986)) infeasible, as they would induce significant unnecessary and hardly quantifiable variance into the flow rates. The general principle of many algorithms is to propagate changes in the exogenously specified (disturbed) flow rate (gradual or discontinuous) to the manipulated flow rate as smoothly as possible, utilising the available free tank volume. In the case of the filter flow rates, the task differs: the natural variance in the buffer tank level should not lead to any adjustment of the flow rates, unless a level peak strictly calls for it; only persistent accumulation of mass should be propagated. Distinguishing one from the other is not easy, and it might even be desirable to postpone a flow rate change until a new cycle is initialised if constant rates are desired in order to truly maximise capacity. Finally, pump power limitations especially at high pressures / cake thickness technically call for some form of constrained control. In the case of the downstream buffer tank, noise effects are less severe due to the absence of filter aid tank refills, especially if cycle times are long. Smooth propagation of accumulating mass to the evaporator could thus be possible, and might furthermore alleviate its tendency to oscillate when the flow rate set point is changed. Still, an algorithm is needed to reliably extract trends form the naturally disturbed level (filter inactivities) to avoid the propagation of naturally occurring noise. Model-predictive level control (Rosander 


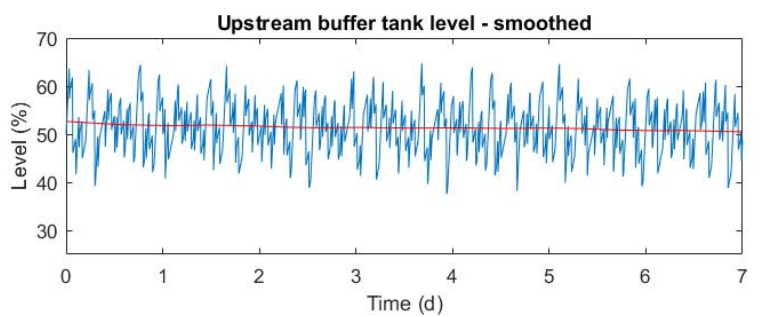

Figure 11: Simulation of buffer tank levels, regarding all regular discontinuities (inactive phases and filter-aid refills, Bähner et al. (2018)) which lead to large process-inherent noise. The red line denotes a smoothed signal, showing that the system is at a steady state, achieved by feed-forward generation of set points (equation (6) )

et al. (2012) ) needs to be mentioned as a means of assuring that buffer tank constraints are respected also in the future while only strictly necessary optimal flow rate changes are induced. However, the absence of a reliable model is limiting at this point.

In general, there seems to be no obvious ready-to-use automation strategy for this type of process. In the case of CP Kelco, the override that utilises the flexibility in the flow rates of the 2 nd coarse filtration to temporarily shift material upstream offers valuable functionality. Still, the traditionally high fill level of the downstream tank should be revised, as it may lead to unnecessary temporary flow rate reductions.

\subsection{Plantwide 6 Local Control Strategy: Conclusions}

It was found that optimal operation of a downstream line in which a battery of parallel, manually reinitialised pressure filters constitute a key element, is everything but trivial. This is largely due the complex dynamics arising from the interaction of discrete and continuous systems at multiple instances in the presence of rapidly moving and changing constraints.

Applying thought concepts from plantwide control research aided in getting a handle on complexity, but academic plantwide control seems to be strongest where process models are available - this is not yet realistic for the pectin plant. It was not possible to determine evident shortcomings in the current operational strategy, but a number of important properties could be pointed out to operators and other stake-holders by analysing historical process data and through plant trials. It is not possible to propose a superior operational structure at this point, but clearly a number of problems could be solved if filter cycles were, to some extent, predictable:

- Facilitated end-time scheduling.

- Earlier capacity estimations (equipment availability).

- Capacity maximisation due to flow increase restriction (initialisation of filters at sustainable flow rates).
- Identifying appropriate filter-aid refill amounts.

Therefore, in the following, a predictive model for the uncertain pressure profiles is sought.

\section{Identification of a Predictive Filtration Model}

Trying to find a model foundation in academic literature is a challenge, as the vast majority of modelling frameworks is developed for the determination of material properties in laboratory trials. Still, as the cake-growth process is quite complex, it was deemed unlikely that an independent ad-hoc model could be found, which is why it was decided to review the existing theoretical knowledge base thoroughly. Among the major modelling frameworks, conventional theory and the compressive rheology approach (Landman \& White (1994); Landman et al. (1995)) should be pointed out. Due to the sparsity of flow rate controlled application scenarios in the latter, conventional theory was deemed the more adequate choice.

\subsection{Conventional Filtration Theory}

Conventional theory builds on the assumptions that Darcy's law governs the flow in all infinitesimal pores, and that the velocity of the solid phase is negligible. Developed to a large extent already in the 1930s, it is well documented, for instance in Tien (2006b). An important milestone for practical considerations was undertaken by Ruth (1935) through the introduction of volume-averaged quantities and the concept of a specific cake resistance, later continued by Grace (1953). This (material-) specific cake resistance is defined as

$$
\alpha=\frac{1}{k \rho_{s} \epsilon_{s}}
$$

where $k$ denotes permeability, and $\epsilon_{s}$ solidosity, the part of a unit volume filled by solid components of density $\rho_{s}$. It allowed a straightforward (often graphical) classification of filter cakes, but already early researchers such as Ruth (1946) noted problems when matching theoretical considerations with industrial practice, originating primarily from operational uncertainties.

\subsection{General Filtration Equation}

Utilising the concept of a cake resistance expression, and after a series of simplifying assumptions (Tien $(2006 \mathrm{~b}))$, the general filtration equation can be obtained:

$$
\frac{d t}{d V}=\alpha \mu \bar{c} \frac{V}{A^{2} \Delta p}+\frac{\mu R_{m}}{A \Delta p}
$$

Here, $R_{m}$ denotes the medium resistance, $V$ the filtered volume of viscosity $\mu$, and $\bar{c}$ the average concentration of retainable solids. $\Delta p$ constitutes the pressure drop corresponding to filtrate flow $d V / d t$. This model is far from being first-principles based, and as pointed out 
decisively e.g. by Tien (2006a), $\alpha$ constitutes a useful fitting-parameter for facilitated experimental analyses of cake properties and ultimately equipment specifications, but has little physical meaning.

\subsubsection{Compressible Filter Cakes}

The assumption of incompressibility is not representative of many filtration processes, and a means of including these effects had to be found. The first heuristic expression was introduced by Sperry \& Baker (1921). The relationship between a material-specific resistance $\alpha_{0}$ and the compressibility affected resistance $\alpha$ over the operational pressure regime becomes:

$$
\alpha=\alpha_{0} p_{l}^{n}
$$

The introduced parameter $n$ is commonly referred to as the 'compressibility index'. It constitutes a further material-specific property which should be determined carefully through experiments. Tiller (1958) deems the compressibility index a decent descriptor for values of $n$ smaller than 0.5-0.7. To amend for a number of formal shortcomings, Tiller \& Leu (1980) introduce the more elaborate power law expression

$$
\alpha=\alpha_{0}\left(1+\frac{p_{s}}{p_{a}}\right)^{n} .
$$

Here, $p_{a}$ is a reference pressure and further parameter. According to Lee \& Wang (2000), equation (10) is more commonly used than equation (9). However, in the establishment of the compressibility-adjusted filtration equation, the pressure gradient through the filter cake requires that an average of the resistance is identified, as indicated in figure 12. For both expressions (eqs. (9) and (10), the averages have been identified in agreement with Durruty (2014), but in the case of expression (10), an explicit reformulation of the filtration equation with respect to pressure drop cannot be identified. As this is a requirement for facile model-implementation in the process control system, expression (9) is used as a basis in the following.

\subsubsection{Negligence of the Medium Resistance}

There is widespread agreement that - in ordinary cake filtration processes - membrane resistance contributes little to the pressure drop. Meindersma et al. (1997) have determined experimentally that the membrane resistance can be neglected for a biological slurry and a perlite body feed. There is more evidence for this simplification, e.g. Soo-Khean et al. (2006). From years of industrial experience, Mayer (2000) comes to the same conclusion. However, the choice of filter medium is attributed significance thinking about e.g. costly ruptures of filter plates and early-stage particle penetration.

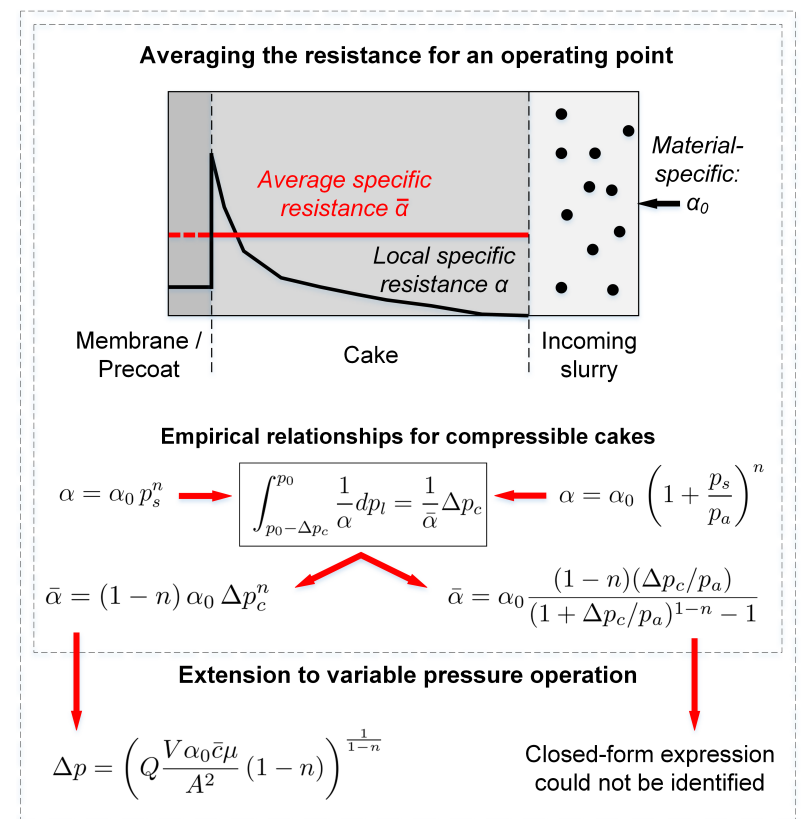

Figure 12: Averaging and extending the descriptiveness of the cake resistance. Successful / unsuccessful derivation of explicit relation for different power-law expressions.

\subsection{Predicting Full-Scale Pressure Cycles}

In the compressibility-adjusted filtration equation (bottom-left of figure 12, , one finds the concentration of solids $\bar{c}$, which in practice cannot be determined on-line. As it is not possible to estimate both $\alpha_{0}$ and $\bar{c}$, the lumped parameter $\alpha_{0, c}^{-}=\alpha_{0} \bar{c}$ is introduced in the final predictor equation:

$$
\Delta p=\left(\frac{Q}{A^{2}}\left(\int_{t_{1}}^{t^{\prime}} Q(t) d t\right) \alpha_{0, c}^{-} \mu(1-n)\right)^{\frac{1}{1-n}}
$$

The parameter estimation process is documented in Bähner et al. (2017a b) and yields a system where the compressibility index $n$ is fixed based on historical data due to correlation properties. The lumped cake resistance $\alpha_{0, c}^{-}$is then identified on-line for each cycle using a moving-horizon estimator based on the most recent $5 \%$ of a cycle profile.

The work at hand furthermore contains a purely statistical predictor. Here, the current capacity estimate is calculated as the average of the last two cycles at the 4 bar threshold. Predictions are then generated based on this value, until a new cycle comes to an end; hereafter the estimate will be updated on all filters. This is illustrated in figure 13 .

\subsubsection{Uncertainty Requirements}

In general, the feasible interval for cleaning grows with the cycle duration: $t_{\text {available }}=t_{f} / N_{u}-t_{c l}$, with $N_{u}$ being the number of units (again, $t_{f}$ denotes the cycle duration). Roughly $10 \%$ of total filtrate (or time) 


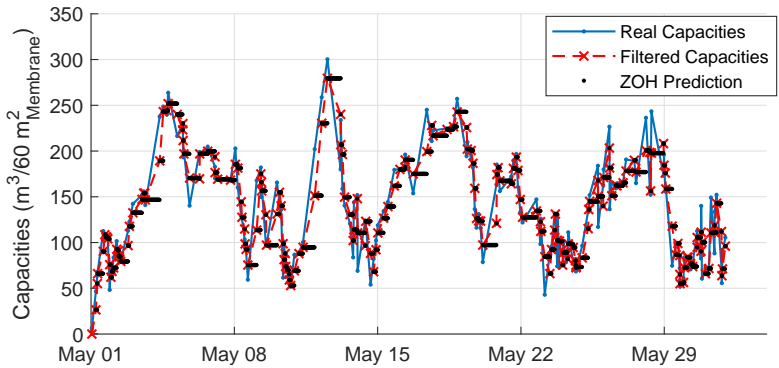

Figure 13: Moving-average filtered cycle capacity prediction, held until a new data-point is generated upon a cycle passing the 4 bar threshold (zero-order hold $(\mathrm{ZOH})$ prediction).

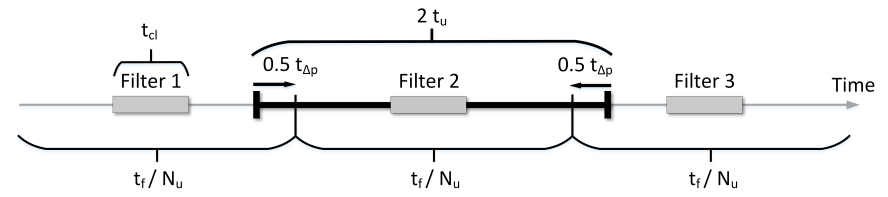

Figure 14: Sketch of uncertainty requirements. Filter cleaning duration (grey bar) in perspective with available time-slot for cleaning and the current final-pressure uncertainty which should be tightened and moved to a higher mean.

correspond to the pressure interval from 4 to 5 bar $t_{\Delta p}=0.1 t_{f}$. Figure $4 \mathrm{p}-2$ shows this interval, where operators take out filters prematurely, expectedly due to scheduling errors or filter-aid shortages. If the uncertainty of the predictions were bounded by $\pm t_{u}$ with $t_{u}=\left(t_{\text {available }}+t_{\Delta p}\right) / 2$, scheduling without flow increases would yield the same take-out pressure distribution. Furthermore, elevating the mean would have a further positive effect on capacity. This is documented in more detail in Bähner et al. (2017b), and a prediction error of $\pm 15 \% 3.5 \mathrm{~h}$ before a cycle ends should leave ample room for scheduling, i.e. by means of flow decreases.
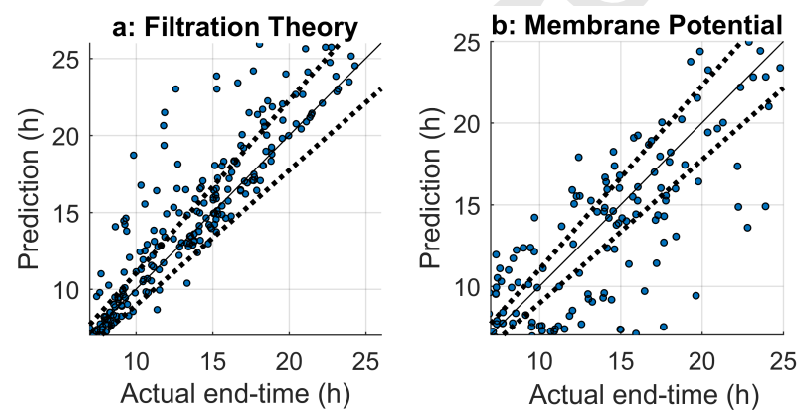

Figure 15: Comparison of filtration theory based predictions (left, Bähner et al. (2017b)) and membrane potential based predictions at 4 bar. Pred. from $3.5 \mathrm{~h}$ before cycle-end, dotted lines denote the prediction accuracy necessary to outperform operators. More samples in the left plot, as not all cycles reach 4 bar.

\subsubsection{Achievable Prediction Accuracy}

The results for the 265 cycles are shown in figure 15. durations of $t_{f}<15 h$ are most relevant for scheduling. The compressibility index $n$ has been tuned such that the prediction error for this cycle length regime is minimised, yielding ca. $0.4-0.5$ for zero-mean predictions. This is well within the bounds discussed by Tiller (1958) as an area where the concept of an averaged specific resistance is meaningful. It implies that, with a tuning-focus on short cycles, long predictions are bound to be skewed, at least given the mathematical framework stemming from classical filtration theory.

Notably, the filtration theory based predictions clearly outperform the purely statistical approach. In the end, the magnitude of the operational uncertainties is too large for a statistical prediction where information from the current cycle is neglected. In a scenario where multiple filters are operated in parallel, and material and especially operational uncertainties are smaller (i.e. by usage of more automated re-initialisation procedures and fewer manual flow rate changes), this approach is still believed to be promising and simple.

Overall, the achievable prediction accuracy fell short of what was expected and hoped-for; likely due to the immense level of fluid- and operational uncertainties. Predictions have also been generated based on the plant trial data, which are more controlled. This showed slightly improved performance, but the sample size is quite small. Furthermore, the predictor needs to be robust enough to be utilised in everyday operations. Lastly, the aptitude of the chosen model still is questionable. There are little to no indicators in academic filtration literature as to which model expression to choose in order to obtain the best predictions.

\section{Conclusion}

In the above, a number of operational challenges were shown that arise when intermittent pressure filters are integrated in a continuous downstream line. These challenges can be derived from the nature of the process, and they have been confirmed in an industrial case study. The control problem is rendered especially interesting, as the actuation strategy can move the capacity constraint. While manual reinitialisation of the filters further complicates the task in the case study plant, the intermittent nature of the filtrations is believed to be a generalisable problem. This holds true also for automatically discharging leaf filters and continuous cross-flow filters which require similar reinitialisation events.

The inherently unsteady buffer tank levels are found to complicate propagating the mass balance by means of automatic level control. Filter cycle prediction based visualisation of the buffer tank level evolution would be a 
valuable tool, but predictions are found to be too imprecise, both for scheduling and inventory control. Mathematically filtering or smoothing the buffer tank level signal in order to visualise trends (depletion or accumulation of material) could aid in their place. However, the (mathematical) filter likely needs to adapt to changing cycle durations, which is all but trivial, and furthermore requires that the necessary advanced functionality is available in the process control system. In general, the application of thought-concepts extracted from plantwide control structure synthesis frameworks aided in organising and understanding the complex scenario at hand, but did not yield improved candidate structures. The alignment of end-times as well as selecting the flow rates that best enable filter capacity while respecting the buffer tank fill constraints - is an inherently complex task, especially without predictions. Prediction precision is impeded by the substantial uncertainties experienced at production scale, which also exacerbate the prediction-free scheduling task. Realistically speaking, a coordinating model-predictive controller would be of need to assure that constraints are not corrupted in the future - but this is not possible due to the lack of a high-fidelity model. It might thus be economically optimal to let operators steer the slowly-evolving plant. Furthermore, a lot of complexity seems to stem from product and peel changeovers. An alignment of production planning procedures to the challenges that arise around the bottleneck 'pressure filtration' might be desirable.

\section{Acknowledgements}

The authors would like to acknowledge the Technical University of Denmark (DTU) and BIOPRO2. This work was supported by the Innovation Fund Denmark through the BIOPRO2 strategic research centre grant number 4105-00020B].

\section{References}

Aske, E. M. B., \& Skogestad, S. (2009). Consistent inventory control. Industrial and Engineering Chemistry Research, 48, 10892-10902. doi 10.1021/ie801603j

Bähner, F., Santacoloma, P., Abildskov, J., \& Huusom, J. (2017a). Analysis and Modelling of an Industrial Pressure Filtration using Process Data. In IFAC-PapersOnLine (pp. 12137-12142). Elsevier volume 50. doi 10.1016/J.IFACOL.2017.08.2152

Bähner, F., Santacoloma, P., Abildskov, J., \& Huusom, J. (2017b). Optimising and Predicting Performance of Industrial Filtrations using Process Data. In Computer Aided Chemical

Engineering (pp. 1471-1476). Elsevier volume 40. doi:10.1016/ B978-0-444-63965-3.50247-6.

Bähner, F. D., Abildskov, J., Huusom, J. K., \& Santacoloma, P. A. (2018). Model-Based Investigation of the Effect of Intermittent Filtration Units on Buffer Tank Levels in a Continuous Process. In Proceedings of the FILTECH 2018 Conference.

Beyer, H., \& Holtzblatt, K. (2010). Contextual Design Chpt. 3 - 4, .

Caroco, R. F., Bevilacqua, M., Armagan, I., Santacoloma, P. A., Abildskov, J., Skov, T., \& Huusom, J. K. (2018). Raw material quality assessment approaches comparison in pectin production. Biotechnology Progress, . doi 10.1002/btpr.2762

Casas-Orozco, D., Villa, A. L., Bustamante, F., \& González, L. M. (2015). Process development and simulation of pectin extraction from orange peels. Food and Bioproducts Processing, 96, 86-98. doi 10.1016/j.fbp.2015.06.006

Durruty, J. (2014). Studies on LignoBoost lignin and the influence of ionic strength. Ph.D. thesis.

Faanes, A., \& Skogestad, S. (2003). Buffer tank design for acceptable control performance. Industrial and Engineering Chemistry Research, 42, 2198-2208. doi 10.1021/ie020525v

Grace, H. (1953). RESISTANCE AND COMPRESSIBILITY OF FILTER CAKES. Chemical Engineering Progress, 49.

Jungbauer, A. (2013). Continuous downstream processing of biopharmaceuticals. Trends in Biotechnology, 31, 479-492. doi 10.1016/j.tibtech.2013.05.011

Landman, K. A., \& White, L. R. (1994). Solid/liquid separation of flocculated suspensions. Advances in Colloid and Interface Science, 51, 175-246. doi 10.1016/0001-8686(94)80036-7.

Landman, K. A., White, L. R., \& Eberl, M. (1995). Pressure filtration of flocculated suspensions. AIChE Journal, 41, 16871700. doi 10.1002/aic. 690410709

Lee, D. J., \& Wang, C. H. (2000). Theories of cake filtration and consolidation and implications to sludge dewatering. Water Research, 34, 1-20. doi 10.1016/S0043-1354(99)00096-2

Luyben, M. L., Tyreus, B. D., \& Luyben, W. L. (1997). Plantwide control design procedure. AIChE Journal, . doi 10.1002/aic. 690431205

Luyben, W. L. (2012). Heuristics for Plantwide Control. In Plantwide Control: Recent Developments and Applications. doi 10.1002/ 9781119968962.ch6

Mannan, S. (2012). Lees' Loss Prevention in the Process Industries: Hazard Identification, Assessment And Control: Fourth Edition. doi 10.1016/C2009-0-24104-3

Mayer, E. (2000). Cake filtration theory and practice. Chemical

engineering journal, 80, 233-236. doi 10.1016/S1383-5866(00) 00095-2

McDonald, K. A., McAvoy, T. J., \& Tits, A. (1986). Optimal averaging level control. AIChE Journal, 32, 75-86. doi 10.1002/ aic. 690320109

Meindersma, G. W., Augeraud, J., \& Vergossen, F. H. P. (1997). Separation of a biocatalyst with ultrafiltration or filtration after bioconversion. Journal of Membrane Science, 125, 333-349. doi 10.1016/S0376-7388(95)00081-X

Murthy Konda, N. V. S. N., Rangaiah, G. P., \& Krishnaswamy, P. R. (2005). Plantwide Control of Industrial Processes: An Integrated Framework of Simulation and Heuristics. Industrial

G Engineering Chemistry Research, 44, 8300-8313. doi 10.1021/ ie $048951 z$

Ochoa, S., Wozny, G., \& Repke, J. U. (2010). Plantwide Optimizing Control of a continuous bioethanol production process. Journal

of Process Control, 20, 983-998. doi 10.1016/j.jprocont.2010. 06.010

Patle, D. S., Ahmad, Z., \& Rangaiah, G. P. (2014). Plantwide Control of Biodiesel Production from Waste Cooking Oil Using Integrated Framework of Simulation and Heuristics. Industrial \&6

Engineering Chemistry Research, 53, 14408-14418. doi:10.1021/ ie 5023699

Price, R. M., \& Georgakis, C. (1993). Plantwide regulatory control design procedure using a tiered framework. Industrial

\& Engineering Chemistry Research, 32, 2693-2705. doi 10.1021/ ie00023a036

Price, R. M., Lyman, P. R., \& Georgakis, C. (1994). Throughput Manipulation in Plantwide Control Structures. Industrial and Engineering Chemistry Research, 33, 1197-1207. doi:10.1021/ ie00029a016

Rathore, A. S., \& Kapoor, G. (2016). Implementation of Quality by Design for processing of food products and biotherapeutics. doi 10.1016/j.fbp.2016.05.009

Rijnsdorp, J. (1991). Integrated Process Control and Automation. Elsevier Science Publishers. 
Rijnsdorp, J. E. (1981). Some cases of human factors in process automation. In Proceedings of the Eighth Triennial World Congress of the International Federation of Automatic Control.

Rosander, P., Isaksson, A., Löfberg, J., \& Forsman, K. (2012). Performance Analysis of Robust Averaging Level Control. In Proceedings of Chemical Process Control VIII.

Rushton, A., Ward, A., \& Holdich, R. (1996). Filtration Process Equipment and Calculations. In Solid-Liquid Filtration and Separation Technology (p. 409).

Ruth, B. (1946). Correlating Filtration Theory with Industrial Practice. Industrial and Engineering Chemistry, 38, 564-571.

Ruth, B. F. (1935). Studies in Filtration III. Derivation of Genera Filtration Equations. Industrial \&6 Engineering Chemistry, 27, 708-723. doi DOI : 10.1021/ie50306a024

Skogestad, S. (2002). Plantwide control: Towards a systematic procedure. Computer Aided Chemical Engineering, 10, 57-69. doi 10.1016/S1570-7946(02) 80039-6

Skogestad, S. (2004). Control structure design for complete chemical plants. Computers and Chemical Engineering, 28, 219-234. doi 10.1016/j.compchemeng.2003.08.002

Skogestad, S. (2012). Economic Plantwide Control. In Plantwide

Control: Recent Developments and Applications. doi:10.1002/ 9781119968962.ch11 arXiv:arXiv:1011.1669v3

Soo-Khean, T., R. B. H., T., \& Tien, C. (2006). Analysis of Cake Filtration DataA Critical Assessment of Conventional Filtration Theory. American Institute of Chemical Engineers AIChE Journal, 52, 3427-3442. doi 10.1002/aic arXiv:arXiv:1402.6991v1

Sperry, D., \& Baker, F. (1921). Notes and Correspondence: A Study of the Fundamental Laws of Filtration Using Plant-Scale Equipment. Industrial \& Engineering Chemistry, 13, 1163-1165. doi 10.1021/ie50144a034

Stephanopoulos, G. (1983). Synthesis of Control Systems for Chemical Plants - A Challenge for Creativity. Computers \& Chemical Engineering, 7, 331-365.

Tarleton, E., \& Hancock, D. (1997). Using Mechatronics for the Interpretation and Modelling of the Pressure Filter Cycle.

Chemical Engineering Research and Design, 75, 298-308. doi 10. $1205 / 026387697523750$

Tarleton, E., \& Willmer, S. (1997). The Effects of Scale and Process Parameters in Cake Filtration. Chemical Engineering Research and Design, 75, 497-507.

Tarleton, E. S. (1998). a New Approach To Variable Pressure Cake Filtration, . I, 53-69. doi 10.1016/S0892-6875(97)00138-6

Tien, C. (2006a). Analysis of Cake Filtration : Volume-Averaged

Continuity. In Introduction to Cake Filtration. doi 10.1016/ B978-0-444-52156-9.50003-5

Tien, C. (2006b). The conventional theory of cake filtration. In Introduction to Cake Filtration (pp. 13-16). doi10.1016/ B978-0-444-52156-9.50002-3.

Tiller, F., \& Leu, W. (1980). Basic data fitting in filtration. J. Chin. Inst. Chem. Eng, 11, 61- 70 .

Tiller, F. M. (1958). The Role of Porosity in Filtration Part 3 Variable-pressure-Variable-rate Filtration. AIChE Journal, 4, 170-174. doi 10.1002/aic.690040210

Van Dijk, M., Dubbelman, S., \& Bongers, P. (2009). Plantwide control of fruit concentrate production volume 7 . IFAC. doi https://doi.org/10.3182/20090712-4-TR-2008.00102

Vasudevan, S., \& Rangaiah, G. P. (2012). A Review of Plantwide Control Methodologies and Applications. In G. P. Rangaiah (Ed.), Plantwide Control: Recent Developments and Applications (pp. 181-97).

Wang, L. (2006). Diatomaceous Earth Precoat Filtration Handbook of Environmental Engineering, Volume 4: Advanced Physicochemical Treatment Processes, 4, 155-189. doi:10.1007/ 978-1-59745-029-4_5

Wu, H., Khan, M., \& Hussain, A. (2007). Process control perspective for process analytical technology: Integration of chemical engineering practice into semiconductor and pharmaceutical industries. Chemical Engineering Communications, 194, 760779. doi 10.1080/00986440601098755
Zheng, A., \& Mahajanam, R. V. (1999). A Quantitative Controllability Index. Industrial \& Engineering Chemistry Research, 38, 999-1006. doi 10.1021/ie980337y 